\title{
fonofone pour iPad et iPhone : cadrage historique et curriculaire d'une application québécoise conçue pour la création sonore en milieu scolaire fonofone for iPad and iPhone: Historical and Curricular Framework for an Application Made in Quebec and Used for Sound Creation in the Classroom
}

\section{Vincent Bouchard-Valentine}

Volume 17, numéro 1, printemps 2016

Apprentissage et enseignement de la musique au $21^{\mathrm{e}}$ siècle : l'apport des sciences et des technologies

URI : https://id.erudit.org/iderudit/1044666ar

DOI : https://doi.org/10.7202/1044666ar

Aller au sommaire du numéro

Éditeur(s)

Société québécoise de recherche en musique

ISSN

1480-1132 (imprimé)

1929-7394 (numérique)

Découvrir la revue

Citer cet article

Bouchard-Valentine, V. (2016). fonofone pour iPad et iPhone : cadrage historique et curriculaire d'une application québécoise conçue pour la création sonore en milieu scolaire. Les Cahiers de la Société québécoise de recherche en musique, 17(1), 11-24. https://doi.org/10.7202/1044666ar

\section{Résumé de l'article}

La création sonore est une forme de création musicale exploitant les qualités expressives du son en dehors des schèmes traditionnels de la mélodie, de l'harmonie et du rythme mesuré. À partir des années 1960, des pédagogues d'origines diverses - Canada, États-Unis, Angleterre et France - ont proposé des modèles d'enseignement fondés sur différentes démarches de création sonore, mais partageant la visée d'une éducation musicale globale et fondamentale. Ces modèles ont été intégrés à partir du tournant des années 1970 dans les programmes d'études scolaires québécois. Toutefois, malgré les efforts déployés au fil des décennies par le ministère de l’Éducation, la création sonore n'est pas véritablement implantée dans les programmes universitaires de formation à l'enseignement de la musique ni dans les pratiques enseignantes en milieu scolaire.

Parmi les outils pédagogiques développés pour la pédagogie de la création sonore, fonofone pour iPad se distingue à double titre. D'une part, il a été spécifiquement conçu pour le milieu scolaire et, d'autre part, sa conception a été pilotée par le compositeur Yves Daoust, une des figures de proue de la musique électroacoustique québécoise.

Cet article a pour objectif général de situer l'interface de création fonofone dans le champ de la pédagogie musicale scolaire. Le design méthodologique fait appel à différentes stratégies de recherche. Pour le volet traitant de l'ancrage pédagogique et curriculaire de l'application, une recherche documentaire (Boisvert 2000) dans le champ de l'éducation musicale a permis de recueillir des données sur la pédagogie de la création sonore, de même que sur la lutherie spécialisée qu'elle a engendrée, en France particulièrement. Pour le volet traitant de l'application fonofone, une entrevue semi-dirigée (Van der Maren 2004) avec le concepteur Yves Daoust a permis de retracer les principaux jalons de son développement et d'en cerner les principales caractéristiques. La consultation des archives personnelles du compositeur québécois et l'observation d'animations réalisées avec l'application en milieu scolaire ont permis de compléter la collecte de données. L'ensemble des données recueillies a été soumis à un processus d'analyse de contenu (Bardin 2007).
Ce document est protégé par la loi sur le droit d'auteur. L’utilisation des services d’Érudit (y compris la reproduction) est assujettie à sa politique d'utilisation que vous pouvez consulter en ligne.

https://apropos.erudit.org/fr/usagers/politique-dutilisation/ 


\title{
fonofone pour iPad et iPhone: Cadrage historique et curriculaire d'une application québécoise conçue pour la création sonore en milieu scolaire
}

\author{
Vincent Bouchard-Valentine \\ (Université du Québec à Montréal)
}

L a création sonore fait partie intégrante des programmes d'études scolaires québécois depuis les années 1970. Toutefois, sur le terrain, le potentiel de cette approche est peu exploité par les enseignants. Parmi les raisons qui expliquent cette situation, il faut noter l'influence de la tradition en pédagogie musicale et le manque de formation des enseignants de musique au regard des stratégies pédagogiques visant le développement chez les élèves de la compétence "Créer-Inventer des œuvres musicales », laquelle figure au Programme de formation de l'école québécoise (Québec 2006).

Depuis une quinzaine d'années, les interfaces de composition numériques opèrent une véritable mutation des pratiques musicales en rendant la création musicale accessible aux amateurs de tout acabit. La popularité des plateformes de partage audio témoigne de cet engouement pour une forme d'expression qui, il y a quelques décennies à peine, exigeait une solide formation musicale et une infrastructure onéreuse lorsque la technologie était impliquée. Quelques centaines de dollars suffisent maintenant pour disposer à son domicile d'un studio semi-professionnel et il n'est plus rare de rencontrer des jeunes ayant développé une pratique de création musicale personnelle en dehors des cadres académiques.

Quiconque s'intéresse un tant soit peu à la technologie musicale connaît la vigueur qui anime ce secteur, tant sur le plan du matériel (hardware) que celui des logiciels (software). L'évolution rapide des technologies musicales de même que la baisse générale de leur coût de revient ouvrent des perspectives inédites pour la pédagogie musicale, particulièrement en milieu scolaire. Il est assez simple aujourd'hui de mettre en place un dispositif technologique permettant à chaque élève d'opérer en classe une station de travail audionumérique individuelle. Un système informatique mobile, une tendance dominante en éducation à l'heure actuelle, rend l'opération encore plus aisée. Parmi les applications musicales disponibles en ce moment pour les tablettes tactiles, fonofone se distingue à double titre. D'une part, il a été spécifiquement conçu pour une pédagogie collective de la création sonore et, d'autre part, sa conception a été pilotée au Québec par le compositeur Yves Daoust, une des figures de proue de la musique électroacoustique québécoise ${ }^{1}$.

Cet article a pour objectif général de situer cet outil pédagogique dans le champ de la pédagogie musicale scolaire. Je présenterai d'abord la notion de création sonore et la place qu'elle a occupé et qu'elle occupe présentement dans le curriculum scolaire québécois. Je situerai ensuite fonofone parmi les applications de création musicale reconnues par le milieu pour leur intérêt pédagogique. Puis, avant de présenter les caractéristiques de l'application, je poserai les jalons de son développement, lequel est ancré dans ce que nous pourrions appeler l'École de lutherie pédagogique française. Je terminerai ce texte en présentant quelques perspectives de développement envisagées par l'équipe technique et pédagogique de fonofone $e^{2}$.

Cet article constitue la première occurrence de fonofone dans une revue scientifique. À cet effet, il convient de mentionner qu'il s'agit d'un premier effort de formalisation d'initiatives essentiellement nourries par une démarche empirique et intuitive. Le design méthodologique prend donc en considération le contexte particulier dans lequel s'inscrit l'objet de la recherche. Ainsi, pour le volet traitant de l'ancrage pédagogique de l'application, j'ai procédé à une recherche documentaire (Boisvert 2000) dans le champ de l'éducation musicale. Pour le volet portant

1 Compositeur prolifique, Yves Daoust (né en 1946) a remporté de nombreux prix au Québec, au Canada et à travers le monde. Fondateur en 1978 de l'Association pour la création et la recherche électroacoustiques du Québec (ACREQ), maintenant connue sous le nom d'ELEKTRA, il a aussi enseigné la composition électroacoustique pendant plus de 30 ans aux Conservatoires de Montréal et de Québec. En 2009, la Fondation ÉmileNelligan lui a décerné le Prix Serge-Garant pour l'ensemble de son œuvre.

2 À noter que l'application est simplement désignée «fonofone» - et non «le fonofone» — sans majuscule. 
sur le développement de l'application, j'ai procédé à une entrevue semi-dirigée (Van der Maren 2004) auprès d'Yves Daoust; j'ai aussi consulté les archives personnelles du compositeur et assisté à des séances d'animation réalisées avec l'application en milieu scolaire. J'ai ensuite procédé à une analyse de contenu qualitative des données recueillies (Bardin 2007).

\section{La pédagogie de la création sonore au Québec}

Au milieu des années 1960, des pédagogues comme R. Murray Schafer (1965) au Canada, Ronald Thomas (1970) aux États-Unis et John Paynter (1970) en Angleterre ont dénoncé le peu de place accordée à la musique contemporaine et à la création dans l'éducation musicale scolaire. Ils ont alors proposé des modèles de creative music priorisant l'expression libre de l'enfant à partir de matériaux sonores divers. La France, grâce notamment aux travaux de Pierre Schaeffer et du Groupe de recherches musicales (GRM), devient rapidement un terreau fertile pour cette approche qui prend l'appellation d'éveil musical. Les compositeurs François Delalande (1984) et Claire Renard (1982), tous deux issus du GRM, en formalisent les principes, largement tributaires de la démarche de composition électroacoustique.

La pédagogie d'éveil-créativité ${ }^{3}$ est essentiellement une transposition en pédagogie musicale du courant expressif en pédagogie des arts plastiques (Lemerise 2006). Il s'agit d'une proposition globale d'éducation musicale actualisant le paradigme de l'enfant-créateur: à travers la démarche de création, l'élève est amené à développer l'ensemble de ses potentialités musicales. La démarche pédagogique est fondée sur le déconditionnement des habitudes d'écoute par la mise en sourdine de toute référence à la musique traditionnelle, qu'il s'agisse des structures mélodico-harmoniques, des schèmes rythmiques ou du code graphique; on parle alors de création sonore pour bien distinguer cette démarche de celle fondée sur la tonalité et le rythme mesuré (qu'on désignera plutôt comme la création musicale). Les exercices et les activités d'apprentissage peuvent, quant à eux, être ramenés à trois types: 1) les élèves se familiarisent avec une notion ou développent une habileté ciblée par l'enseignant à travers des exercices ou jeux musicaux; 2) les élèves explorent différentes idées musicales ou procédés de composition en réalisant des improvisations collectives; 3) les élèves s'engagent dans un projet de création sonore individuel ou collectif qui pourra éventuellement être interprété devant public (Bouchard-Valentine 2016b).

Au Québec, la pédagogie d'éveil-créativité est introduite dans les programmes scolaires dès la fin des années 1960, alors que la société québécoise est engagée dans ce qu'on désigne comme la Révolution tranquille. Deux ouvrages fondateurs publiés à cette époque redéfiniront les visées de l'éducation musicale. Le rapport Parent (1964), issu de la Commission royale d'enquête sur l'enseignement dans la province de Québec, et le rapport Rioux (1969), issu de la Commission d'enquête sur l'enseignement des arts au Québec, reconnaissent de façon claire et explicite la valeur fondamentale de l'éducation artistique. Le rapport Rioux, en particulier, propose un projet de société duquel l'art et l'éducation artistique sont partie intégrante (Couture et Lemerise 1992). L'éducation artistique doit préparer l'individu à vivre dans un monde en perpétuelle transformation: la familiarisation à l'art contemporain à travers une pédagogie ouverte permet à l'élève d'apprendre à vivre avec l'imprévisible et de cheminer d'un état de déséquilibre vers un état d'équilibre (Rioux 1969, 19-20). La musique y est d'ailleurs présentée comme une organisation sonore - première référence explicite à la notion de création sonore dans un document officiel - à travers laquelle l'élève se développe: «[i]l ne faut pas lui imposer des schèmes sonores traditionnels, mais éduquer sa spontanéité dans l'expression par le son» (Rioux 1969, 27-28).

Les programmes d'études en musique publiés à la fin des années 1960 concrétisent les orientations des rapports Parent et Rioux. Ces documents, appelés programmescadres (Québec 1969, 1970), sont concis et se limitent à exposer l'esprit, les objectifs et les contenus pour chacune des disciplines. Ils marquent cependant une étape importante pour l'éducation musicale, puisqu'ils proposent une formation musicale globale visant le développement des capacités d'expression et de créativité par le chant et la pratique instrumentale. Précisons que les programmes du département de l'Instruction publique qu'ils remplacent se limitaient au chant religieux ou profane et aux rudiments du solfège (Allard et Lefebvre 1998). Les nouveaux programmes-cadres préconisent les méthodes actives européennes - Orff, Martenot, Kodály, Suzuki - pour les niveaux primaire et secondaire. L'expression libre et la création sonore figurent aussi parmi les énoncés d'intentions pédagogiques de ces programmes, mais leurs modalités de mises en œuvre ne sont pas précisées. Il faut attendre la publication du guide Expression musicale à l'élémentaire (Québec 1976) pour qu'elles soient explicitées. Elles s'appuient sur les propositions issues de la creative music anglo-saxonne et de l'éveil musical français.

Dix ans après le rapport Parent, un réexamen du système d'éducation s'impose. La publication du Livre vert sur l'enseignement primaire et secondaire (Québec 1977) et l'organisation de vastes consultations publiques permettent d'en tirer un bilan. À la suite de cet exercice,

\footnotetext{
Claude Dauphin (2011) utilise la contraction de «creative music» et «d'éveil musical» pour désigner ce courant pédagogique.
} 
le gouvernement publie L'école québécoise: Énoncé de politique et plan d'action (Québec 1979) dans lequel il définit les objectifs et les orientations d'une relance du système d'éducation axée sur la qualité de l'enseignement. Les programmes de musique découlant de cette réforme donnent clairement la priorité à la création sonore (Québec 1981a, 1981b). La musique y est présentée comme «une succession de sons et de silences choisis et organisés par des individus (compositeurs), avec des buts expressifs, et en vue d'être écoutée » (Québec 1981a, 3), et l'approche pédagogique privilégiée met l'emphase sur une démarche de création centrée sur «l'exploitation et l'organisation de solutions sonores par l'enfant»(Québec 1981a, 3). Bien conscients du changement de posture qu'ils exigent des enseignants, les concepteurs de ces programmes ajoutent une note d'avertissement:

La lecture de ce document peut troubler certains enseignants en provoquant diverses remises en question, soit par la présentation d'une hiérarchie différente des valeurs musicales, soit par la proposition de langages musicaux nouveaux et d'une approche pédagogique axée sur la création (Québec 1981a, 105).

Ces programmes d'études sont accompagnés de guides pédagogiques abondamment illustrés d'exemples pour orienter les enseignants (Québec 1982a, 1982b, 1983). Cette série de guides est complétée en 1990 par le document $L a$ création sonore au secondaire: Comment faire parler les sons! (Québec 1990).

Dans les années 1990, comme c'est le cas dans plusieurs pays occidentaux, le Québec s'engage dans un processus de réforme éducationnelle pour adapter son système d'éducation aux caractéristiques et aux besoins d'une société en transformation. Les programmes de musique issus de la Politique éducative (Québec 1997) adoptent une position d'équilibre ou de complémentarité entre l'approche fondée sur la tonalité et celle fondée sur le son. Nous trouvons donc au fil des pages de ces programmes des références aux deux approches sans parti pris pour l'une ou pour l'autre. Notons cependant que le Domaine des arts est caractérisé par des savoirs essentiels communs aux quatre disciplines artistiques, lesquels sont associés à la démarche de création.

Malgré les efforts déployés par le ministère de l'Éducation pour valoriser les approches créatives depuis le début des années 1970, la création sonore demeure rare en milieu scolaire. D'ailleurs, une étude de 2011 a révélé que les enseignants de musique consacrent moins de 20 pour cent du temps d'enseignement au développement de la compétence «Créer-Inventer», toutes approches confondues (PierreVaillancourt et Peters 2013). L'éducation musicale en milieu scolaire reste, encore aujourd'hui, fortement ancrée dans le paradigme de l'enfant-interprète. Plusieurs facteurs peuvent expliquer cette situation, mais l'influence de la tradition pédagogique et le manque de formation des enseignants de musique sont à placer en tête de liste. Il se trouve que, depuis le $\mathrm{XIX}^{\mathrm{e}}$ siècle, la formation des musiciens est essentiellement orientée vers l'interprétation vocale ou instrumentale. Les habiletés d'improvisation ne font pas partie de la formation courante - sauf pour le jazz - et la composition est habituellement enseignée comme une spécialité aux niveaux supérieurs (Després et Dubé 2012). Il s'ensuit qu'une forte proportion des formateurs d'enseignants et des étudiants inscrits aux programmes universitaires de formation initiale à l'enseignement sont peu familiers avec les démarches de création en musique. Par ailleurs, les recherches québécoises portant sur la pédagogie de la création musicale en milieu scolaire sont rares et celles portant sur la pédagogie de la création sonore sont pratiquement inexistantes ${ }^{4}$. Un autre facteur pourrait contribuer à la faible part impartie à la création sonore dans les pratiques enseignantes: le manque de ressources didactiques. En effet, les ouvrages de référence de la creative music et de l'éveil musical sont fondés sur un cadre théorique et conceptuel issu des années 1960 et 1970. Malgré la richesse des idées qu'ils renferment, ces écrits n'ont pas été traduits et révisés en fonction du développement des champs de savoirs de l'éducation, de l'éducation artistique et de l'éducation musicale. Dans le contexte de professionnalisation de l'enseignement qui est le nôtre, l'enseignant est appelé à concevoir lui-même des activités d'enseignement-apprentissage selon une approche par compétences (Québec 2006). Or, la plupart des enseignants n'ont ni le temps ni l'expertise pour s'approprier et transposer le contenu de ces ouvrages trop souvent évasifs sur des éléments de planification élémentaires: désignation des objectifs et des contenus d'apprentissage, progression des apprentissages, formules pédagogiques, stratégies d'enseignement, d'apprentissage et d'évaluation.

En dépit d'une apparente désaffection du milieu scolaire pour la création sonore, il importe néanmoins de rappeler que l'éducation musicale est, plus que jamais, concernée par le sonore. En effet, sous l'impulsion initiale des avant-gardes artistiques du début du $\mathrm{Xx}^{\mathrm{e}}$ siècle, la ligne de démarcation entre musique et environnement sonore s'est graduellement estompée (Solomos 2013). Dans ce qu'il est convenu d'appeler aujourd'hui les arts sonores, musiciens et artistes explorent, analysent et interrogent les multiples rapports entre son, musique et environnement (Bosseur 2016). Pour un groupe toujours croissant de musiciens, l'environnement sonore est même devenu le terrain d'investigation privilégié,

\footnotetext{
4 Notons toutefois au passage les publications pédagogiques de Robert Léonard (1981, 1995) et le document Faire parler les sons, publié par le Musée de la civilisation (Goupil et Bellavance 1992).
} 
fournissant l'essentiel du matériau compositionnel. Sur le fond, donc, l'objet de l'éducation musicale contemporaine englobe un ensemble de pratiques que l'on qualifiait autrefois de bruitistes en raison de leur caractère expérimental, mais qui sont aujourd'hui couramment intégrées aux musiques populaires et savantes.

\section{La création musicale sur iPad}

L'apparition, il y a une quinzaine d'années, d'interfaces numériques de création musicale abordables, au visuel attrayant, à l'ergonomie intuitive et aux possibilités en apparence illimitées a renouvelé l'intérêt des chercheurs pour la création musicale en milieu scolaire. Ces interfaces, prenant la forme de stations audionumériques, d'instruments virtuels ou simplement de jeux interactifs, pourraient bien changer la répartition du temps consacré à la création en classe de musique : certains prédisent même un changement paradigmatique (Demski 2011). Ce mouvement vers la création musicale pourrait s'accélérer à mesure que les interfaces gagnent en simplicité et en portabilité. Les tablettes tactiles suscitent d'ailleurs depuis quelques années un engouement technologique sans précédent en éducation (Karsenti et Fievez 2013) comme dans plusieurs autres secteurs d'activité. La musique n'y échappe pas et le répertoire des applications musicales croît à une vitesse exponentielle.

Dans une recherche récente (Bouchard-Valentine 2015), j'ai voulu savoir quelles applications étaient réputées appropriées pour le développement des compétences de création musicale en contexte scolaire. L'objectif de cette recherche était de produire une typologie des applications de création musicale pour tablettes tactiles à partir de palmarès créés par des professionnels en enseignement de la musique. Compte tenu des contraintes temporelles et financières, j'avais limité mon analyse aux applications compatibles avec iOS 9 pour iPad, technologie qui supporte la majorité des applications musicales disponibles. J'ai analysé près de 70 applications que j'ai classées dans sept catégories (éditeurs de partitions, instruments, séquenceurs, logiciels de traitement audionumériques, mélangeurs, enregistreuses et banques de sons). Un classement supplémentaire selon le niveau de complexité de l'application été effectué pour en estimer le public scolaire cible: les applications pour jeunes enfants qui visent les élèves d'âge préscolaire, les applications pour grand public qui visent les élèves d'âge primaire et secondaire, et les applications spécialisées qui s'adressent aux musiciens semi-professionnels ou professionnels.

Les applications les plus fréquemment citées pour la création musicale en milieu scolaire sont les séquenceurs à boucles tels que GarageBand ou Auxy. Ces applications font appel à un séquenceur permettant d'agencer des boucles audio préenregistrées qui forment des séquences musicales d'une qualité étonnante et qui donnent à l'apprenti musicien le sentiment de composer sa propre musique. Cependant, à l'instar des logiciels de correction linguistique, ces logiciels prennent en charge les opérations qui exigent une maîtrise de la grammaire musicale. Les paramètres de l'organisation sonore (tonalité, harmonie, tempo, rythme, mélodie) sont prédéterminés afin que les différentes boucles puissent s'agencer harmonieusement. Par ailleurs, la palette stylistique mise à la disposition des élèves se limite le plus souvent à la musique pop et à ses déclinaisons. La conception même de ces applications confine alors les élèves dans une esthétique musicale standardisée: une musique tonale de type pop, le plus souvent soutenue par une section rythmique prononcée et structurée par groupes de 16 mesures. Bien que ces interfaces soient très appréciées des élèves et qu'elles présentent un intérêt pédagogique certain, la créativité musicale qu'elles mettent en œuvre apparaît plutôt restreinte au regard des visées d'une éducation artistique fondée sur la dynamique de création et sur l'élargissement des horizons culturels préconisés par le ministère de l'Éducation (Québec 2006).

En outre, ces populaires séquenceurs à boucles ont d'abord été conçus pour une utilisation individuelle. Leur emploi en situation collective implique une division du groupe en îlots individuels, en dyade ou en petites équipes autour d'un appareil. Peu importe le mode d'organisation choisi, un seul élève à la fois peut manipuler la tablette. Lorsque les élèves travaillent seuls ou en petites équipes, des modalités particulières d'organisation de la classe doivent être prises pour éviter la congestion sonore occasionnée par les multiples démarches musicales engagées simultanément. À la fin d'une activité de création, la pièce musicale peut être sauvegardée en fichier audio et écoutée avec un lecteur multimédia; les élèves n'interviennent pas dans l'exécution de leur pièce. Enfin, ces applications sont essentiellement des dérivés simplifiés de logiciels conçus au départ pour des musiciens professionnels. Elles n'ont pas été développées dans une optique pédagogique et sont essentiellement utilisées en milieu scolaire en raison de leur facilité d'emploi, de la qualité des résultats musicaux obtenus et du sentiment de compétence qu'elles procurent. L'enseignant doit alors faire preuve de jugement afin de les utiliser dans une perspective d'apprentissage.

Les applications de type synthétiseurs ou qui se limitent à reproduire des instruments existants sont légion. Elles permettent de former un orchestre numérique ou mixte avec les instruments traditionnels présents dans la classe. La question de la démarche pédagogique reste cependant entière puisque la tablette tactile prend ici le rôle d'un instrument parmi d'autres. La série de treize applications SmartFaust 
pour téléphone intelligent fait partie de cette catégorie. Développées par le Centre national de création musicale (2016) chacune des applications SmartFaust permet de transformer le profil d'un son, préalablement programmé par l'utilisateur, en déplaçant le téléphone dans l'espace. Ces applications sont notamment utilisées avec des groupes d'élèves pour des concerts de musique électronique.

La recherche que j'ai effectuée a mis en évidence la rareté des applications pédagogiques de création musicale collective. À ma connaissance, la seule application spécifiquement conçue pour le développement de la compétence à créer des œuvres musicales est fonofone de Cosimu $^{5,6}$.

\section{Les appareils précurseurs}

fonofone présente un ensemble de caractéristiques sans équivalent dans le marché actuel des applications pour tablettes tactiles. Toutefois, la conception de cette application peut être rattachée à l'École française de lutherie pédagogique et plusieurs des principes qui ont balisé son développement sont hérités d'expériences réalisées depuis le début des années 1970 par différents organismes engagés dans la diffusion et la médiation des pratiques de création sonore auprès du jeune public.

Rappelons que le mouvement d'éveil musical en France découle en grande partie des travaux de Pierre Schaeffer, inventeur avec Pierre Henry de ce qu'ils ont appelé la musique concrète, travaux qui se sont poursuivis au sein du GRM. La démarche compositionnelle concrète rompt avec la tradition musicale occidentale fondée sur la représentation abstraite des sons au moyen d'une notation symbolique. Elle s'appuie sur un processus dialogique de recherche d'expressivité où alternent, dans un mouvement continu, des opérations de transformation et d'appréciation des sons. La musique émerge progressivement de ce modelage de la matière sonore, par explorations successives (Schaeffer 1966).

La transposition de cette démarche en pédagogie musicale implique que les apprentissages soient effectués à travers des activités d'exploration, d'improvisation et de création sonore dans lesquelles le développement des habiletés d'écoute occupe la place centrale. Il s'agit essentiellement d'amener les élèves du sonore au musical (Céleste, Delalande et Dumaurier 1982) en leur permettant, par l'application d'une démarche de création fondée sur l'exploration et le tâtonnement, d'expérimenter et de ressentir différents rapports de tension entre les sons. Le but poursuivi est l'affinement de la sensibilité aux propriétés des sons et aux organisations sonores. En cela, il s'agit bien d'une éducation esthétique (Reimer 2003). En contexte scolaire, les activités pédagogiques respectent le caractère collectif de la situation pédagogique en engageant simultanément l'ensemble du groupe dans une réalisation commune, tout en favorisant l'expression de la créativité individuelle.

L'éveil musical s'est développé en France suivant trois écoles de pensée distinctes, mais complémentaires. Ces différentes postures peuvent aujourd'hui être fédérées au sein d'une approche globale prônant la diversité des modes d'expression.

La première école de pensée repose sur les sons humains. Guy Reibel et ses Jeux musicaux (Reibel 1984; Reibel et Heyligers 2006) en est certainement le plus éminent représentant. La voix et le corps offrent ici un large éventail de possibilités sonores à exploiter en situation de création sonore collective.

La seconde école privilégie l'emploi de corps sonores acoustiques. Tout objet est ici susceptible de produire des sonorités utilisables dans une création sonore. Les objets du quotidien sont prisés pour leur accessibilité, leur faible coût et la diversité des sonorités qu'ils peuvent produire (Delalande 1984). Dans certains cas, les élèves sont amenés à concevoir leurs propres instruments en s'inspirant des lutheries acoustiques expérimentales (Vandervost 2006). Toujours en France, les frères Baschet, pour leur part, ont conçu un instrumentarium pédagogique composé de 14 structures sonores adaptées à la taille d'élèves du primaire (Association structures sonores Baschet [ASSB] 2016). Cet instrumentarium incarne l'idéal de Bernard Baschet, ingénieur au GRM, d'une exploration sonore réalisée avec des moyens purement acoustiques, sans électricité (Bouchard-Valentine 2016a).

La troisième école exploite les technologies électriques, électroniques et numériques ${ }^{7}$. Au départ, le matériel technologique se limitait aux outils électroacoustiques courants pour l'enregistrement, l'écoute, la transformation et le montage des sons sur un support matériel: microphones, magnétophones et haut-parleurs. Des appareils électroniques ont ensuite été développés par des studios indépendants pour des actions pédagogiques en milieu scolaire ou extrascolaire (Veitl 2001). Trois de ces appareils méritent ici une brève

\footnotetext{
5 Cosimu est un organisme sans but lucratif qui regroupe les concepteurs de l'application fonofone. Sa mission: développer des outils pédagogiques pour l'initiation à la création sonore et coordonner des activités de formation dans les milieux scolaire et extrascolaire (Cosimu [2017]. fonofone, www.fonofone.com, consulté le 24 janvier 2018).

6 [NDLR: On pourra lire, dans le présent numéro des Cahiers, l'article d'Eldad Tsabary portant sur une autre application de la technologie dans un contexte de création musicale au niveau universitaire («Stabilizing and Destabilizing Agents in Laptop Orchestra Improvisation»).]

7 Je me réfère principalement pour cette section à l'étude réalisée par Anne Veilt en 2001, qui demeure la plus complète à ce jour. J'ai complété et mis à jour l'information pour les besoins de cette synthèse.
} 
description: le Gmebogosse, l'UPIC et le Mélisson. Ces appareils ont marqué leur époque et, par la suite, les plus intéressantes de leurs caractéristiques ont été incorporées dans le Musicolateur, puis fonofone, par Daoust et Burton.

\section{Le Gmebogosse (1973)}

Le Gmebogosse a été développé par le Groupe de musique expérimentale de Bourges $\left(\mathrm{GMEB}^{8}\right)$ et mis en service en 1973 pour des animations en milieu scolaire. L'appareil a évolué entre 1973 et 2008 suivant sept versions différentes. À partir de la sixième version, au tournant des années 2000, l'appareil adopte la technologie numérique et devient le Cybersongosse (Institut de musique électroacoustique de Bourges [IMEB] 2016).

Le Gmebogosse-Cybersongosse était constitué d'un ensemble de consoles branchées à un dispositif de diffusion et reliées entre elles pour former une chaîne de traitement électroacoustique. Chaque console pouvait accueillir deux élèves et comprenait un ensemble très complet d'outils pour capter, produire, transformer et diffuser les sons. La console était divisée en zones - capteurs, sources, synthèse, traitements, spatialisation, enregistrement, montage, mixage - qui regroupaient les différents outils: séquenceurs, oscillateurs, filtres, égalisateur, automatisation, etc. (IMEB 2016) La manipulation de l'appareil ne demandait pas de connaissances ou de dextérité particulières (Veitl 2001).

Un cahier pédagogique d'environ 300 jeux a été élaboré au fil des ans. Ce cahier était accompagné d'une bibliothèque de sons - au départ enregistrés individuellement sur cassettes audio - qui servaient à alimenter l'appareil. L'ensemble, par sa simplicité d'opération, permettait le jeu collectif en direct (c'est-à-dire en temps réel) et favorisait le travail collaboratif d'écoute (IMEB 2016).

Le Gmebogosse a connu une certaine renommée en France et à l'international, mais l'encombrement et la lourdeur des consoles, de même que le temps requis avant chaque séance pour le paramétrer en fonction des jeux à réaliser ont grandement nui à sa diffusion (Veitl 2001).

\section{L’UPIC (1977)}

L'Unité polyagogique informatique (UPIC) a été lancée en 1977 par l'équipe du Centre d'études de mathématique et automatique musicales (CEMAMu, rebaptisé Centre Iannis Xenakis [CIX] en 2006). Née du rêve de Xenakis de mettre au point un appareil qui libèrerait le compositeur de la notation musicale traditionnelle, l'UPIC opère une correspondance entre le dessin, la synthèse sonore et la musique (CIX 2016). Cet appareil a été pensé pour démocratiser l'accès à la composition musicale. L'idée de convertir les traits de crayons en modulations sonores est très riche sur le plan pédagogique, mais la taille et le coût du dispositif rendent son implantation improbable en milieu scolaire. De plus, le design de l'interface impose un mode de jeu individuel ou en groupes très restreints (Nelson 1997), ce qui pose des défis importants pour la gestion collective de la classe. Une nouvelle version de l'UPIC est en développement depuis 2013.

\section{Le Mélisson (1983)}

Le Mélisson a été présenté en 1983 par le Groupe de musique électroacoustique d'Albi-Tarn (GMEA) et est toujours en service (GMEA 2016). Il est composé d'un ensemble de boîtiers indépendants qui peuvent être reliés entre eux pour former un synthétiseur modulaire analogique. La configuration du synthétiseur est établie en fonction des besoins pédagogiques et des boîtiers disponibles. Un ensemble de base pour le travail scolaire comprend 40 boîtiers dont les couleurs diffèrent selon les fonctions : les boîtiers rouges correspondent aux générateurs de signaux, les verts aux générateurs d'enveloppes, les jaunes aux filtres, les bleus aux mélangeurs et les noirs à l'alimentation électrique (GMEA 2016).

L'idée derrière cette modularité est de permettre à chaque élève de disposer d'un boîtier simple à manipuler afin qu'il puisse participer à la création collective. La complexité de l'ensemble est donc ramenée pour chaque élève à quelques boutons. Veitl (2001) fait remarquer que la simplicité excessive du Mélisson finit par limiter le potentiel d'intervention de chaque élève. De plus, le Mélisson est restreint aux sons électroniques et ne permet pas la captation de sons naturels, comme c'est le cas pour le Gmebogosse par exemple.

\section{Le Musicolateur (20059)}

Au début des années 1970, à la suite de ses études en composition au Conservatoire de musique de Montréal, Yves Daoust entreprend un stage de composition électroacoustique au GMEB, alors en voie de devenir l'un des pôles majeurs de la musique électroacoustique européenne. En quelques années, les compositeurs Françoise Barrière et Christian Clozier, assistés de quelques collaborateurs, ont réussi à y fonder un studio, à mettre sur pied en 1971 le festival international Synthèse et à lancer en 1973 le Concours international des musiques de créations électroniques de Bourges. On y avait également développé le Gmebogosse, comme mentionné plus haut, un dispositif électronique pour initier les enfants à la création électroacoustique. Dans le cadre de son stage, Daoust a eu l'occasion

\footnotetext{
8 En 1994, le GMEB devient l'Institut de musique électroacoustique de Bourges (IMEB) et poursuit ses activités jusqu'à sa dissolution en 2011.

9 Cette section s'appuie sur une entrevue d'Yves Daoust réalisée par l'auteur à l'été 2016 et sur l'analyse de ses archives personnelles.
} 
d'assister à des animations centrées sur cet instrument dans les écoles de la campagne environnante. Il est alors fasciné par l'enthousiasme des élèves et la spontanéité avec laquelle ils s'investissent dans les propositions de création électroacoustique.

À son retour au Québec, il s'engage dans la promotion de la musique électroacoustique. Il fonde en 1978 avec Philippe Ménard (né en 1946) et un groupe de compositeurs l'Association pour la création et la recherche électroacoustiques du Québec $\left(\mathrm{ACREQ}{ }^{10}\right)$. Cherchant divers moyens de faire connaître ce type de musique à différents publics, Daoust et Ménard envisagent même la possibilité d'acquérir un Gmebogosse pour des actions pédagogiques en milieu scolaire. L'appareil est cependant trop dispendieux pour la petite association.

Ce projet tomba dans l'oubli jusqu'à ce que la Société de musique contemporaine du Québec (SMCQ) approche Daoust en 2002 pour la création d'une œu vre interactive ayant pour but d'initier les enfants à la musique contemporaine. S'associant à l'artiste pluridisciplinaire Alexandre Burton, Yves Daoust s'oriente plutôt vers la conception d'un instrument d'initiation à la création sonore assez simple pour être manipulé par des enfants d'âge préscolaire, mais offrant des possibilités sonores suffisamment riches pour intéresser les musiciens adultes. Une subvention du Conseil des arts du Canada leur permet d'imaginer un instrument entièrement nouveau, reposant sur des concepts inédits et une technologie de pointe, permettant de travailler les sons aussi simplement et spontanément que de dessiner avec un crayon sur une feuille de papier, dans l'esprit de l'UPIC imaginé par Xenakis. La petite équipe, à laquelle s'est ajouté Glen Silver pour l'élaboration des interfaces numériques, et l'artiste visuel Daniel Sylvestre pour le design de l'instrument, consacrera trois ans à la fabrication du Musicolateur qui sera finalement lancé en 2005.

Le Musicolateur est un ensemble de huit pupitres munis chacun de quatre plaques tactiles, divisées en zones, représentant chacune un mode de travail sur le son, de sculpture sonore, si l'on veut. Ces pupitres sont reliés à un ordinateur qui contient le logiciel et les sons préprogrammés. Les plaques sont donc des contrôleurs qui envoient à l'ordinateur un signal numérique dont la valeur correspond à des coordonnées cartésiennes (abscisse et ordonnée). L'ordinateur interprète cette valeur et l'attribue à une fonction programmée dans le logiciel Max/MSP. Les pupitres sont branchés à des interfaces sonores et à une console virtuelle, laquelle permet l'amplification par haut-parleurs de l'ensemble du dispositif. Chaque pupitre dispose en plus d'une interface audio permettant aux élèves d'entendre ce qu'ils font dans des écouteurs.

Lors des animations pédagogiques, les élèves se regroupent par deux ou même par quatre autour des pupitres. Ils sont amenés à inventer des sons et à les articuler collectivement dans une forme musicale à travers un processus d'écoute mutuelle. Le Musicolateur est donc à la fois un outil de création et un instrument de jeu collectif. Il peut être considéré comme un instrument de musique de chambre pouvant être intégré à différents types de performance: musique mixte, théâtre, poésie, etc.

Ces animations sont progressivement confiées à Félix Boisvert, compositeur et ancien étudiant d'Yves Daoust au Conservatoire de musique de Montréal. Entre 2005 et 2013, près de 1000 ateliers ont été donnés en milieu scolaire, communautaire ou dans le cadre d'événements organisés par la SMCQ. Des projets pédagogiques en partenariat avec des ensembles professionnels ont également été réalisés, notamment avec IMusici de Montréal, l'Orchestre symphonique de Longueuil et le quatuor de saxophones Quasar.

Au tournant de 2010, le Musicolateur arrive au terme de sa durée de vie utile. Il était, de toute manière, condamné à disparaître à plus ou moins brève échéance, car il s'agissait d'un produit artisanal dont le coût de reproduction était prohibitif. L'équipe ne disposant que d'un seul ensemble de pupitres, il était difficile d'envisager un accroissement des activités de formation. Par ailleurs, le fonctionnement de l'appareil nécessitait le déploiement d'une logistique trop importante: il fallait un camion pour le déplacer et prévoir au moins 45 minutes pour le monter dans une classe.

\section{fonofone $^{11}$}

L'avènement des tablettes tactiles permet à Yves Daoust et à Alexandre Burton d'envisager la continuité du projet Musicolateur sous une autre forme. L'informatique mobile résout enfin le problème d'encombrement commun à tous les dispositifs jusqu'alors développés pour la création sonore en milieu scolaire, en plus d'offrir un potentiel de rayonnement à l'international. À partir de la fin de 2011, Daoust et Burton s'attèlent à la réalisation d'un prototype de l'application (version alpha) pour explorer la faisabilité du projet. Le cahier des charges vise la conception d'une interface intuitive et facile à utiliser dans le cadre d'une approche collective de la création sonore. L'application doit aussi permettre la performance en direct afin que les

\footnotetext{
10 L'association existe toujours aujourd'hui, mais se fait davantage connaître à travers l'événement Elektra, «un festival international d'art numérique présentant des œuvres et des artistes qui allient art et nouvelles technologies» (Festival Elektra [2015]. ELEKTRA — Mission, http://elektramontreal. ca/fr/elektra mission.html, consulté le 24 janvier 2018).

11 Cette section s'appuie sur une entrevue d'Yves Daoust réalisée par l'auteur à l'été 2016 et sur l'analyse de ses archives personnelles.
} 
élèves puissent compléter le cycle complet d'une démarche musicale: création, interprétation et appréciation. Enfin, l'application doit comporter un certain nombre de fonctions permettant à l'enseignant de concevoir et d'archiver des activités pédagogiques que les élèves pourraient ensuite activer sur leur tablette. Cette phase exploratoire ayant été complétée avec succès, un deuxième prototype (version bêta) est lancé en 2013 pour fin d'expérimentation avec des élèves. Des animations pédagogiques sont organisées avec ce nouvel outil dans la continuité des actions déjà réalisées avec le Musicolateur.

En 2015, l'organisme Cosimu fait appel à la compagnie Inookta pour programmer une version commercialisable de l'application, laquelle est lancée sur l'App Store le 22 février 2017. Deux versions sont alors proposées: une version gratuite limitée dans ses fonctions et une version payante offrant toutes les fonctionnalités. L'application commerciale vise également le marché de l'utilisateur individuel (amateur ou professionnel) avec une fonction multipiste qui permet de superposer les sons afin d'enrichir les possibilités compositionnelles. L'élève ayant découvert l'application en classe peut ainsi continuer son activité créatrice à la maison.

\section{Les caractéristiques de l'application}

fonofone est à la fois un mini studio de création électroacoustique et un instrument numérique permettant à des groupes d'élèves de s'engager dans une démarche de création sonore et de jeu collectif en direct. En cela, fonofone est un instrument du $\mathrm{XxI}^{\mathrm{e}}$ siècle qui s'inscrit dans la grande tradition de la pédagogie de musique d'ensemble. Les tablettes peuvent être branchées à un système de diffusion sonore centralisé, mais lors des animations en milieu scolaire, chaque tablette est habituellement reliée par câble audio à un petit haut-parleur que l'élève porte autour du cou. Il est également possible d'utiliser fonofone en mode individuel, même en situation scolaire. Dans ce cas, les élèves se concentrent sur leur propre démarche à l'aide d'un casque d'écoute.

Prenant appui sur la démarche compositionnelle des électroacousticiens, les élèves de tous âges sont amenés à explorer et à transformer les sons à l'aide des outils de l'application, puis à élaborer ensemble une œuvre musicale qui sera interprétée collectivement en direct. L'interface propose donc un ensemble d'outils de traitement du son représentés par des formes géométriques simples et colorées. Ainsi, aucun mot n'apparaît sur la surface de jeu (Figure 1). Le mouvement des doigts sur ces formes active le traitement du son et permet de le moduler. Le choix des formes est le fruit de plusieurs années de réflexion et d'expérimentation avec le Musicolateur et fonofone; par exemple, l'utilisation du triangle pour le fragmenteur découle de la nécessité d'offrir,
Figure 1: L'interface de l'application fonofone.

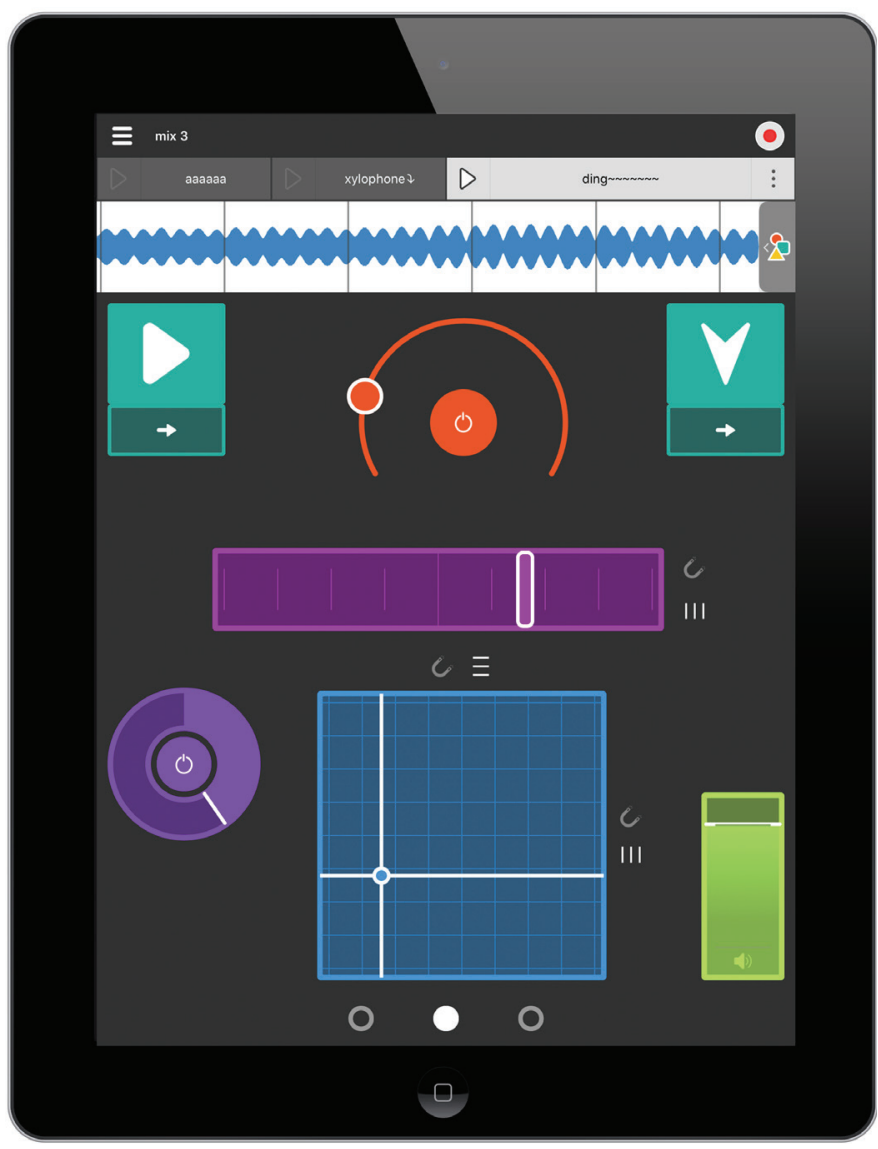

pour cet outil spécifique, un continuum d'options allant du blocage complet (haut) jusqu'à une multitude de possibilités (bas). Le Tableau 1 (page suivante) explique les différentes fonctions liées aux différentes formes géométriques.

Les concepteurs de cette interface graphique visaient trois objectifs : 1) une utilisation intuitive des outils permettant de saisir spontanément la relation de cause à effet entre le mouvement des doigts et le rendu sonore; 2) une utilisation transversale de l'application depuis le préscolaire jusqu'à la fin du secondaire; 3) une diffusion internationale sans barrière linguistique.

Comme pour le Gmebogosse et le Mélisson - qui ont été conçus dans une perspective éducative - fonofone peut être paramétré en fonction d'une intention pédagogique qui fait appel à certains types de sons ou d'outils. Une activité pour débutants pourrait ainsi se limiter à un bruit blanc et à l'outil volume: la consigne pourrait être de reproduire le son des vagues s'échouant sur une plage.

La liste des outils apparaît dans un ruban situé dans la partie supérieure de l'écran. Les outils désirés peuvent être sélectionnés et déposés à l'endroit souhaité sur la surface de jeu. Il est possible de télécharger jusqu'à trois sons et de les superposer si on le désire. L'enseignant peut aussi 
Tableau 1 : Les outils de l'interface de fonofone.

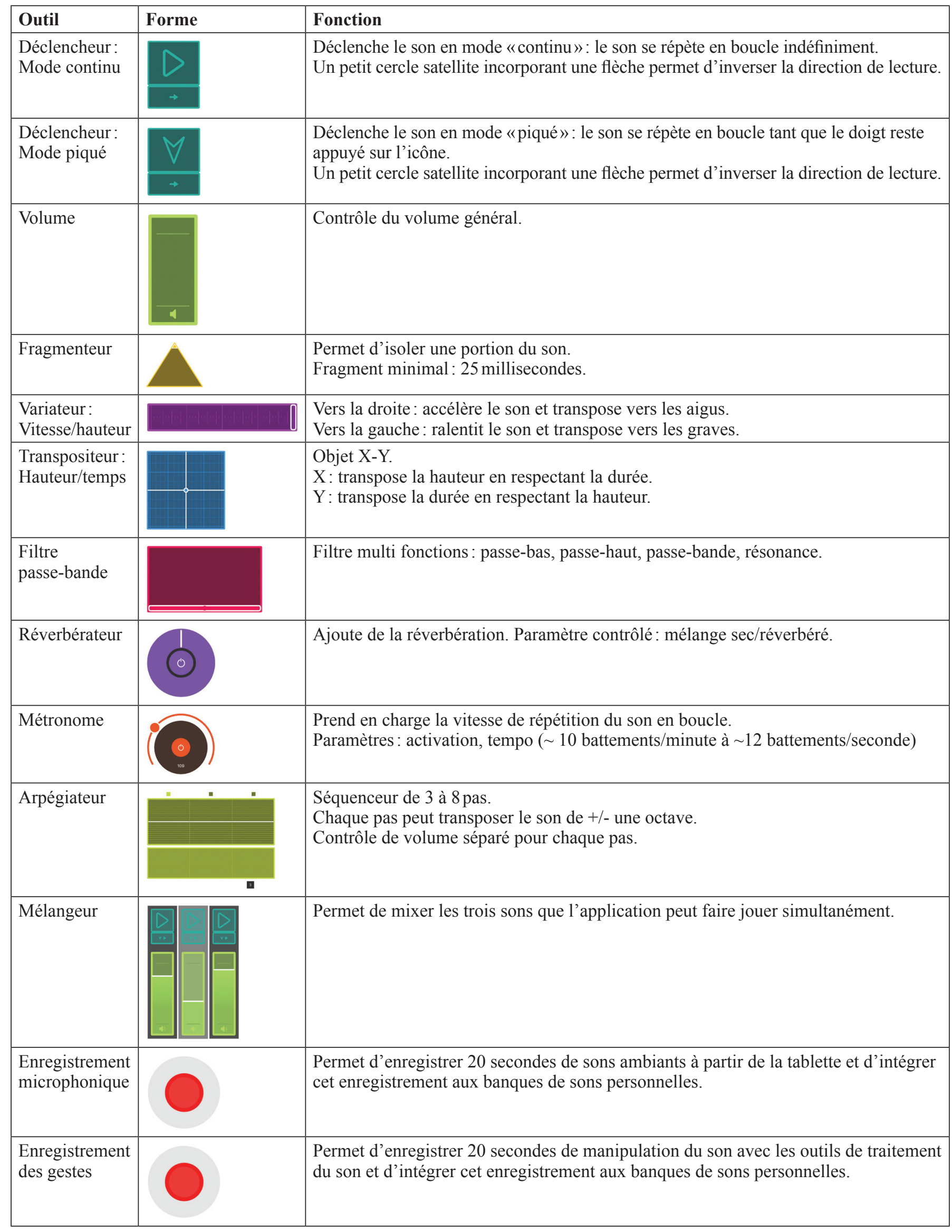


créer une activité et la sauvegarder. Dans ce cas, les élèves n'ont qu'à sélectionner l'activité souhaitée parmi celles qui sont affichées sur leur tablette pour débuter la situation d'enseignement-apprentissage. Au fil du temps, l'enseignant peut se constituer une bibliothèque d'activités réutilisables et adaptées aux besoins de formation de ses élèves. L'application offre ici une modularité et des possibilités expressives inégalées pour un dispositif pédagogique.

\section{L'approche pédagogique}

Les animations réalisées jusqu'à présent avec le Musicolateur et fonofone ont été ponctuelles, échelonnées sur quelques heures pour les ateliers ou sur quelques semaines pour les résidences. Elles sont donc orientées vers l'initiation, visent un premier contact avec la démarche de création sonore. La pédagogie développée par Yves Daoust et mise en œuvre par l'équipe d'animation de fonofone tire son inspiration du séjour que Daoust a effectué à Bourges dans les années 1970. La notion de jeu y occupe une place centrale, de même que l'idée de mettre l'élève le plus rapidement possible en interaction avec la matière sonore. Les activités doivent être stimulantes pour les élèves, tout en étant pertinentes sur les plans pédagogique et musical. Le principe de la pédagogie active prend ici toute sa mesure:

Dès le départ, avant même d'enseigner quoi que ce soit, je les mets en situation d'explorer les différents outils - les premières explorations sont d'ailleurs totalement ouvertes. Ensuite, je leur pose des questions: «Le truc vert sur l'écran, il sert à quoi ?» Ils ne possèdent évidemment pas les notions musicales, mais ils sont capables de décrire l'effet de l'outil sur le son, l'effet de ralentissement par exemple. Je les amène à exprimer, dans leurs propres mots, la manière dont ils ont ressenti la transformation du son, éprouvé la charge affective du traitement sonore. Dans leurs propos, je décèle immédiatement leur préférence pour tel type d'effet ou tel degré d'effet. Nous commençons alors à explorer les possibilités expressives du son avec les différents outils; c'est à partir de ce moment que nous entrons véritablement dans l'univers musical. À travers les différentes activités, nous précisons le vocabulaire, nous abordons l'acoustique, nous expérimentons différents procédés de composition et nous illustrons le tout avec des exemples puisés dans le répertoire classique ou contemporain. Quand tous ces éléments sont arrimés - «Créer, Interpréter, Apprécier» -, cette démarche d'éducation musicale devient extrêmement riche (Daoust, entrevue semi-dirigée, 15 juin 2016).

Trois pôles ou degrés d'intervention sont à distinguer dans l'approche de Daoust: les exercices ou «jeux sonores», les inventions et les créations libres.

\section{L'exercice}

L'exercice vise le développement de l'écoute analytique et la compréhension des outils. Par exemple, le jeu intitulé $L e$ son caché fait entendre un son de quelques secondes dans lequel une petite incongruité a été introduite, un élément qui ne devrait pas se trouver là. Les élèves doivent la repérer à l'oreille et, ensuite l'isoler avec le fragmenteur. De tels exercices ne durent que quelques minutes et n'occupent qu'une dizaine de minutes par séance. Ils permettent d'explorer les différents paramètres du son (timbre, hauteur, durée et intensité), de développer des habiletés de discrimination auditive, de même que la dextérité nécessaire à la manipulation de certains outils. L'interface étant très simple à utiliser, les élèves peuvent se concentrer sur l'écoute du rendu sonore. Les techniques expérimentées dans les exercices peuvent ensuite être réinvesties dans une invention.

\section{L'invention}

L'invention est une activité de création collective très ciblée, circonscrite à une technique, une idée ou un geste qui sera exploré et approfondi. La proposition de création est amenée par l'enseignant, mais elle est nourrie par les élèves. L'enseignant anime la démarche de création, amène les élèves à s'impliquer et fixe les critères de réussite. Par exemple, après avoir exploré le variateur de vitesse dans un exercice, les élèves sont amenés à créer une accumulation. Les élèves doivent déterminer comment, à partir de sons ponctuels, ils arriveront à créer l'effet d'une pluie fine qui s'amplifierait jusqu'au déluge. Pour aiguiller les élèves, l'enseignant leur fait entendre différents exemples d'accumulations puisés dans le répertoire classique ou contemporain. Une fois les idées trouvées, le groupe les met à l'essai et s'exerce pour obtenir l'effet escompté. Ces inventions amènent les élèves à expérimenter différents procédés de structuration sonore qui leur serviront à mettre la musique en forme dans la création libre.

\section{La création libre}

La création libre est complètement ouverte et fait appel à ce qu'Yves Daoust nomme «l'imaginaire sans limites» (Daoust, entrevue semi-dirigée, 15 juin 2016). Lorsque les élèves ont acquis un certain bagage de connaissances, développé un ensemble d'habiletés et constitué un répertoire d'idées musicales, ils sont prêts à s'engager dans un projet de création sonore collective. Ce projet les amènera à concevoir et à élaborer une pièce musicale originale qu'ils pourront interpréter, par exemple, devant un public. Ces créations libres peuvent être réalisées en grands groupes ou en groupes restreints. Au-delà de la réalisation d'une pièce musicale, les élèves sont aussi engagés dans une démarche musicale 
globale: ils sont amenés à produire des partitions musicales pour consigner et développer leurs idées, à interpréter euxmêmes leur création sonore et, enfin, à apprécier, selon des critères définis, leurs réalisations ou celles des autres élèves. Au regard des orientations du Programme de formation de l'école québécoise, ils développent les trois compétences disciplinaires du programme de musique.

\section{Quelques perspectives de développement}

Le lancement de fonofone sur l'App Store le 22 février 2017 pourrait marquer le coup d'envoi d'une véritable diffusion de l'application dans le milieu scolaire québécois, mais aussi - et peut-être surtout - à l'international. La création sonore demeure encore aujourd'hui une niche pédagogique spécialisée, mais bien vivante dans certains milieux répartis dans différents pays, comme le projet CREAMUS (France) ou le projetEars2 (Royaume-Unis). La constitution d'un réseau international de praticiens et de chercheurs pourra sans doute contribuer à développer et à renforcer ce type d'expertise. Il n'est pas question de remplacer l'objet traditionnel de l'enseignement musical, mais bien de tenir compte, dans un esprit de complémentarité des approches, de l'évolution des pratiques musicales et du développement global de l'élève à travers celui des compétences musicales, particulièrement celles liées à la création musicale.

Derrière la conception du Musicolateur, devenu par la suite fonofone, se cache la volonté d'Yves Daoust d'offrir au public une clé d'accès aux pratiques musicales contemporaines. L'ensemble de la carrière d'Yves Daoust est d'ailleurs marqué par une forme de " missionnariat» esthétique, que ce soit comme cofondateur de l'ACREQ en 1976 (association dont il a été le directeur artistique de 1983 à 1987 et de 1989 à 1991), comme professeur de composition aux Conservatoires de musique de Québec et de Montréal de 1980 à 2011, ou comme compositeur de musique pour jeune public.

Il demeure que la pierre angulaire d'une réelle implantation des musiques contemporaines dans le milieu scolaire passe par la formation des enseignants, puisque ce sont eux qui sont appelés à les intégrer aux activités ordinaires de la classe. Actuellement, la majorité des enseignants de musique ne possèdent pas la formation nécessaire pour exploiter fonofone de manière optimale. Le problème ne se situe pas tant au niveau des compétences informatiques que sur les plans esthétique et pédagogique. Daoust en est bien conscient et c'est pourquoi l'application fonofone est indissociable d'une plateforme pédagogique en ligne, actuellement en développement. À terme, cette plateforme inclura une zone sécurisée donnant accès à un ensemble de ressources pour les enseignants et les élèves (tutoriels et formation en ligne, répertoire de jeux sonores et d'inventions, banques de sons, etc.), un espace de consignation pour les réalisations des élèves, ainsi qu'un forum d'échanges. Les enseignants de musique qui ne sont pas familiers avec l'approche pédagogique trouveront sur cette plateforme des modules d'autoformation qui leur permettront de s'approprier les démarches d'enseignement et d'apprentissage qui la fondent.

Aucune expérience n'ayant été menée en milieu scolaire sur une période de plus d'une année, nous ne disposons pas encore d'exemple de progression pédagogique au terme de laquelle les élèves seraient en mesure de démontrer un niveau de maîtrise élevé des trois compétences disciplinaires («Créer-Inventer, Interpréter, Apprécier»). Même si l'approche pédagogique s'appuie sur la spontanéité et l'expression de l'élève, elle implique la construction de savoirs, savoir-faire et savoir-être qui restent pour l'instant à expliciter et à formaliser dans un modèle d'enseignement. Une subvention de recherche de l'Université du Québec à Montréal (PARFARC, volet 1) m'a permis d'entreprendre avec Yves Daoust la structuration de cette plateforme et la formalisation d'un modèle pédagogique.

Dans son analyse critique des technologies pédagogiques pour la création sonore, Veitl souligne que «l'intérêt d'un outil réside non pas dans le nombre élevé des fonctionnalités proposées, mais dans les différentes utilisations (y compris les plus inattendues pour ses concepteurs) auxquelles il peut se prêter» $(2001,36)$. fonofone recèle un fort potentiel de développement. Actuellement compatible avec la plateforme iOS 11, pour iPad et iPhone, fonofone pourrait être programmé pour la plateforme Android et employé sur d'autres types d'interfaces, comme les tableaux numériques interactifs ou les téléphones intelligents. L'utilisation de capteurs de mouvements qui activeraient les différents outils de traitement permettrait d'ajouter une dimension corporelle, dans l'esprit d'une pédagogie du geste musical préconisée par Claire Renard (1982) ou de l'approche interdisciplinaire décrite dans les ouvrages de Magali Viallefond (1992). Ces ramifications de l'application originale s'étendront suivant la disponibilité des ressources financières et la nature des projets qui verront le jour. Enfin, la création d'œuvres pour fonofone qui se révèleraient intéressantes sur le plan musical et adaptées aux besoins de formation des élèves pourrait être une occasion pour les compositeurs de réinvestir le milieu scolaire.

\section{Conclusion}

fonofone de Cosimu est le dernier né d'une lignée d'appareils développés depuis le début des années 1970 pour la pédagogie collective de la création sonore. Par rapport à ces prédécesseurs, cette application iPad opère un bond technologique et ergonomique prodigieux qui laisse 
espérer un meilleur enracinement des pratiques de création sonore en milieu scolaire. À l'état de prototype jusqu'à tout récemment, son rayonnement dans le milieu scolaire québécois est demeuré confidentiel. Le lancement d'une première version commercialisée sur l'App Store devrait favoriser l'émergence d'initiatives porteuses à l'échelle internationale. Toutefois, une réelle implantation dans le milieu scolaire suppose une meilleure formation des enseignants de musique sur l'aspect de la création sonore. L'équipe de fonofone se penchera au cours des prochaines années sur l'élaboration de la plateforme interactive en ligne et sur l'établissement d'un réseau international de praticiens et de chercheurs.

\section{RÉFÉRENCES}

Allard, Michel et Bernard Lefebvre (1998). Les programmes d'études catholiques francophones: Des origines à aujourd'hui, Montréal, Logiques.

Association structures sonores Baschet (ASSB), http:// www.baschet.org/, consulté le 5 novembre 2017.

BARDIN, Laurence (2007). L'analyse de contenu, Paris, Presses universitaires de France.

BOISVERT, Danielle (2000). « Recherche documentaire et informationnelle », dans Thierry Karsenti et Lorraine Savoie-Zajc (dir.), Introduction à la recherche enéducation, Sherbrooke, Université de Sherbrooke, p. 57-77.

Bosseur, Jean-Yves (2016). Musique et environnement, Paris, Minerve.

BOUCHARD-VALENTINE, Vincent (2015). Interfaces numériques de création musicale: Une typologie des applications pour tablettes tactiles, communication présentée le 5 novembre dans le cadre de Learning and Teaching Music in the Twenty-First Century: The Contribution of Science and Technology, McGill University et Université du Québec à Montréal, Montréal.

Bouchard-Valentine, Vincent (2016a). «Genèse de l'instrumentarium Baschet: 14 structures sonores pour une pédagogie de la création sonore», dans Marco A. T. Nascimento et Adeline Stervinou (dir.), Ensino e Aprendizagens Musicais no Mundo: Formação, diversidade e currículo com ênfase na formação humana. Anais $2^{\circ}$ Conferência Internacional de Educação Musical de Sobral, de 22 à 25 de julho de 2015, Sobral (Brésil), Universidad Federal de Ceará, p. 72-80.

Bouchard-Valentine, Vincent (2016b). «Trajetória da creação sonora no curriculo escolar quebequence », dans Marco A. T. Nascimento et Adeline Stervinou (dir.), Ensino e Aprendizagens Musicais no Mundo, Sobral (Brésil), Sobral Gráfica e Editora.
CÉleste, Bernadette, François Delalande et Élisabeth Dumaurier (1982). L'enfant du sonore au musical, Paris et Bry-sur-Marne, Buschet/Chastel et Institut national de l'audiovisuel.

Centre Iannis Xenakis (CIX). Centre Iannis Xenakis, http:// www.centre-iannis-xenakis.org/, consulté le 5 novembre 2017.

Couture, Francine et Suzanne Lemerise (1992). «Le Rapport Rioux et les pratiques innovatrices en arts plastiques», dans Léon Bernier et collab. (dir.), Hommage à Marcel Rioux: Sociologie critique, création artistique et société contemporaine, Montréal, Albert Saint-Martin, p. 77-94.

DAUPHIN, Claude (2011). Pourquoi enseigner la musique?: Propos sur l'éducation musicale à la lumière de l'histoire, de la philosophie et de l'esthétique, Montréal, Presses de l'Université de Montréal.

Delalande, François (1984). La musique est un jeu d'enfant, Paris et Bry-sur-Marne, Buschet/Chastel et Institut national de l'audiovisuel.

Demski, Jennifer (2011). «Music Instruction Goes Digital », The Education Digest, vol. 76, $\mathrm{n}^{\circ}$ 7, p. 14-18.

DesPrés, Jean-Philippe et Francis Dubé (2012). «Proposition d'un cadre conceptuel pour aider le professeur d'instrument à intégrer l'improvisation musicale à son acte pédagogique», Intersections: Canadian Journal of Music, vol. 32, $\mathrm{n}^{\circ} 1$ et 2, p. 143-165.

Groupe de musique électroacoustique d'Albi-Tarn (GMEA). GMEA, http://www.gmea.net/, consulté le 5 novembre 2017.

Goupil, Louise et Carole Bellavance (1992), Faire parler les sons, Québec, Musée de la civilisation, Service de l'éducation, 1 vidéocassette.

Centre national de création musicale (GRAME). Accueil GRAME, http://www.grame.fr/, consulté le 5 novembre 2017.

Institut de musique électroacoustique de Bourges (IMEB). http://www.imeb.net/, consulté le 30 septembre 2016.

Karsenti, Thierry et Aurélien Fievez (2013). L'iPad à l'école, usages, avantages et défis: Étude auprès de 6057 élèves et 302 enseignants du Québec. Rapport préliminaire des principaux résultats, Montréal, Centre de recherche interuniversitaire sur la formation et la profession enseignante (CRIFPE).

LEMERISE, Suzanne (2006). Du dessin aux arts plastiques: L'héritage moderniste d'Irène Senécal, Québec, Presses de l'Université du Québec.

LÉONARD, Robert (1981). 14 jeux sonores du GRAM, Montréal, Université de Montréal. 
LÉONARD, Robert (1995). Jeux sonores pour voix et petits instruments de percussion, Verdun, Musigraphe et UMMUS.

Nelson, Peter (1997). « The UPIC System as an Instrument of Learning », Organised Sound, vol. 2, nº 1, p. 35-42.

Parent, Alphonse-Marie (1964). Rapport Parent: Rapport de la Commission royale d'enquête sur l'enseignement dans la province de Québec. Les structures pédagogiques du système scolaire: Les programmes d'études et les services éducatifs, vol. 2b, Québec, Commission royale d'enquête sur l'enseignement dans la province de Québec.

Paynter, John et Peter Aston (1970). Sound and Silence: Classroom Projects in Creative Music, Cambridge, Cambridge University Press.

Pierre-Vaillancourt, Zara et Valerie Peters (2013). «Le programme de formation de l'école québécoise: Résultats préliminaires du sondage FAMEQ 2011», Musique et pédagogie, vol. 28, $\mathrm{n}^{\circ}$ 1, automne, p. 21-23.

Québec (1969).Programme d'études des écoles secondaires. Arts: Éducation musicale: 112131415112223242 521323334353 54, Québec, Ministère de l'Éducation, Direction générale de l'enseignement élémentaire et secondaire, Service des programmes.

Québec (1970). Programme d'études des écoles élémentaires. Arts: Expression musicale, Québec, Ministère de l'Éducation, Direction générale de l'enseignement élémentaire et secondaire, Divison des beaux-arts.

Québec (1976). Art: Expression musicale à l'élémentaire: Guide pédagogique, Découverte des sons, Québec, Éditeur officiel du Québec.

Québec (1977). L'enseignement primaire et secondaire au Québec: Livre vert, Québec, Ministère de l'Éducation.

Québec (1979). L'école québécoise : Énoncé de politique et plan d'action, Québec, Ministère de l'Éducation, Service général des communications.

Québec (1981a). Programme d'études: Primaire. Art: Art dramatique, arts plastiques, danse, musique, Québec, Ministère de l'Éducation.

Québec (1981b). Programme d'études: Secondaire, musique, Québec, Ministère de l'Éducation, Direction générale du développement pédagogique, Direction des programmes, Service du secondaire.

Québec (1982a). Guide pédagogique: Primaire. Musique: Premier cycle, Québec, Ministère de l'Éducation, Direction générale du développement pédagogique, Direction des programmes, Service du primaire.
Québec (1982b). Guide pédagogique: Primaire. Musique: Second cycle, Québec, Ministère de l'Éducation, Direction générale du développement pédagogique, Direction des programmes, Service du primaire.

Québec (1983). Guide pédagogique: Secondaire. Musique, Québec, Ministère de l'Éducation, Direction générale du développement pédagogique, Direction des programmes, Service du secondaire.

Québec (1990). La création sonore au secondaire: Comment faire parler les sons! Activités réalisées par des enseignantes et des enseignants des 11 régions du Québec, Québec, Ministère de l'Éducation.

Québec (1997). L'école, tout un programme: Énoncé de politique éducative, Québec, Ministère de l'Éducation.

Québec (2006). Programme de formation de l'école québécoise: Éducation préscolaire, enseignement primaire, Québec, Ministère de l'Éducation, du Loisir et du Sport.

Reibel, Guy (1984). Jeux musicaux: Essai sur l'invention musicale, vol. 1: «Jeux vocaux», Paris, Salabert.

Reibel, Guy et Béatrice Heyligers (2006). Le jeu vocal: Chant spontané, Paris, MK2, 1 disque vidéo digital.

ReIMER, Bennett (2003). A Philosophy of Music Education: Advancing the Vision, $3^{\mathrm{e}}$ édition, Upper Saddle River, Prentice Hall.

Renard, Claire (1982). Le geste musical, Paris/Fondettes, Hachette/Van de Velde.

Rioux, Marcel (1969). Rapport de la Commission d'enquête sur l'enseignement des arts au Québec, vol. 2, Québec, Éditeur officiel du Québec.

Schafer, Raymond Murray (1965). The Composer in the Classroom, Toronto, BMI Canada.

SCHAEFFER, Pierre (1966). Traité des objets musicaux, Paris, Seuil.

Solomos, Makis (2013). De la musique au son: L'émergence $d u$ son dans la musique des XXe et XXI siècles, Rennes, Presses universitaires de Rennes.

Thomas, Ronald B. (1970). Manhattanville Music Curriculum Program. Final report, Washington, D.C., United States Office of Education, Bureau of research.

VAN DeR MAREn, Jean-Marie (2004). Méthodes de recherche pour l'éducation, $2^{\mathrm{e}}$ édition, Bruxelles, De Boeck.

Vandervost, Max (2006). Nouvelles lutheries sauvages, Paris, Gallimard.

VeItL, Anne (2001). Quelles ressources technologiques pour renouveler les pédagogies de la musique? Présentation critique d'outils. Rapport d'enquête, Paris, Ministère de la Culture, Direction de la musique, de la danse, du théâtre et des spectacles. 
Viallefond, Magali (1992). Formation d'éveil musical1:

Le corps, Vanves, Ministère de l'Éducation nationale, de l'Enseignement supérieur et de la Recherche, Centre national d'enseignement à distance de Vanves. 


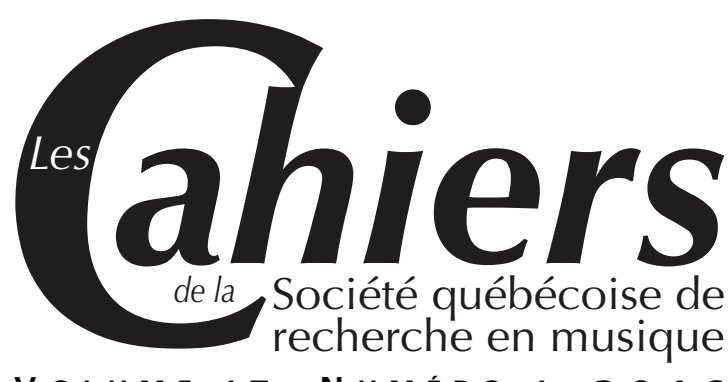

VOLUME 17 - N U M É R $1-2016$

\section{Apprentissage et enseignement de la musique au $21^{e}$ siècle : L'apport des sciences et des technologies}

\section{AU SOMMAIRE DE CE NUMÉRO}

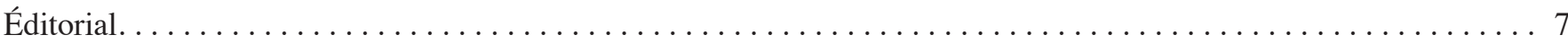

Isabelle Cossette et Isabelle Héroux

fonofone pour iPad et iPhone: Cadrage historique et curriculaire d'une application québécoise $\ldots \ldots \ldots \ldots \ldots . \ldots 11$ conçue pour la création sonore en milieu scolaire

Vincent Bouchard-Valentine

Des logiciels audio-vidéo à l'enseignement de l'éducation musicale dans l'enseignement secondaire français

Pascal Terrien

Stabilizing and Destabilizing Agents in Laptop Orchestra Improvisation

Eldad Tsabary

A 3D Camera User Interface for Wrist Angle Monitoring in Piano Performances

Jennifer MacRitchie and Christopher Baylis

L'intégration de sites web d'hébergement de vidéos dans l'enseignement de l'instrument: Usages et

pratiques pédagogiques

Jérôme A. Schumacher

La simulation de concours d'orchestre: Analyse qualitative et située de l'activité des musiciens

Roberta Antonini Philippe et Angelika Güsewell 


\section{COMPTES RENDUS}

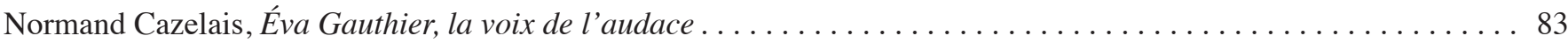

Marie-Thérèse Lefebvre

Jonathan Goldman (dir.), La création musicale au Québec . . . . . . . . . . . . . . . . . . . . . . . . . . . . 89

Ariane Couture

Fiona Magowan, Louise Wrazen (dir.), Performing Gender, Place, and Emotion in Music. Global Perspectives . . . 92 Bruno Deschênes, Catherine Harrison-Boisvert

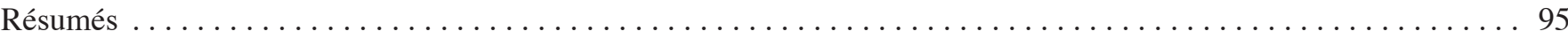

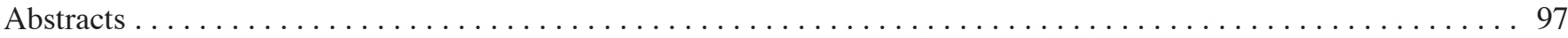

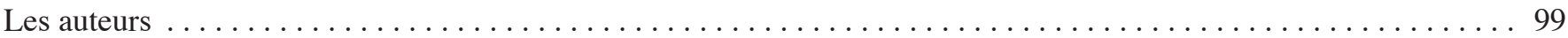

NOTES

Les chercheurs désirant proposer un article aux Cahiers de la Société québécoise de recherche en musique sont invités à communiquer avec le rédacteur en chef de la revue, Jean Boivin (Jean.Boivin@USherbrooke.ca), avant de soumettre leur article. Pour tout autre renseignement, veuillez-vous référer au protocole de rédaction, disponible sur le site Internet de la Société québécoise de recherche en musique (SQRM): www.sqrm.qc.ca.

La revue est distribuée gratuitement aux membres de la SQRM via la plateforme électronique Érudit. Pour devenir membre, veuillez compléter le formulaire d'adhésion disponible sur le site Internet de la SQRM. Les non-membres désirant s'abonner à la revue peuvent contacter Érudit (https://www .erudit.org/).

Pour se procurer un numéro d'archives en version papier (volumes 1 à 12), il faut contacter la direction administrative de la SQRM à info@sqrm.qc.ca.

La revue est financée par le Fonds de recherche du Québec - Société et culture (programme Soutien aux revues scientifiques) et est produite par la Société québécoise de recherche en musique.

Adresse postale: $\quad$ Société québécoise de recherche en musique

Département de musique de l'Université du Québec à Montréal

Case postale 8888, succursale Centre-ville

Montréal (Québec) H3C 3P8

Adresse physique: Département de musique de 1'Université du Québec à Montréal

1440, rue Saint-Denis, local F-4485

Montréal (Québec) H2X 3J8

Téléphone : 514-987-3000, poste 4075

info@sqrm.qc.ca

Avant d'être publié, chaque texte fait l'objet d'une évaluation de la part du comité scientifique et de relecteurs externes.

Les opinions exprimées dans les articles publiés par Les Cahiers de la Société québécoise de recherche en musique n'engagent que leurs auteurs.

Société québécoise de recherche en musique, 2016

Dépôt légal : Bibliothèque nationale du Québec et

Bibliothèque nationale du Canada

ISSN 1480-1132 (Imprimé)

ISSN 1929-7394 (En ligne)

ISBN 978-2-924803-12-7

(C) Les Cahiers de la Société québécoise de recherche en musique, Printemps 2016, Copyright 2018

Tous droits réservés pour tous les pays. 